\title{
Nonthermal and thermal diagnostics of a solar flare observed with RESIK and RHESSI
}

\author{
E. Dzifčáková ${ }^{1,2}$, A. Kulinováa ${ }^{1,2}$, C. Chifor ${ }^{3}$, H. E. Mason ${ }^{3}$, G. Del Zanna ${ }^{4}$, J. Sylwester $^{5}$, and B. Sylwester ${ }^{5}$ \\ 1 Astronomical Institute Academy of Sciences of the Czech Republic, 25165 Ondřejov, Czech Republic \\ e-mail: [elena;kulinova] @asu.cas.cz \\ 2 Department of Astronomy, Physics of the Earth and Meteorology, Faculty of Mathematics Physics and Informatics, \\ Comenius University, Mlynská dolina, 84248 Bratislava, Slovakia \\ e-mail: [dzifcakova; kulinova]@fmph.uniba.sk \\ 3 Department of Applied Mathematics and Theoretical Physics, Centre for Mathematical Sciences, Wilberforce Road, \\ Cambridge CB3 0WA, UK \\ e-mail: [c.chifor;h.e.mason]@damtp.cam.ac.uk \\ 4 Mullard Space Science Laboratory, University College London, Holmbury St. Mary, Dorking, Surrey RH5 6NT, UK \\ e-mail: gdz@mssl.ucl.ac.uk \\ 5 Space Research Centre, Polish Academy of Sciences, 51-622, Kopernika 11, Wroclaw, Poland \\ e-mail: [js;bs]@cbk.pan.wroc.pl
}

Received 27 July 2007 / Accepted 31 May 2008

\section{ABSTRACT}

\begin{abstract}
Aims. We aim to prove and diagnose the occurrence of nonthermal electron distributions in solar flare plasma using X-ray spectral observations.

Methods. An M4.9 flare on 2003 January 7/8 was observed with the RESIK instrument in the 3-6 ^ wavelength range (2-4 keV) and with RHESSI at energies above $6 \mathrm{keV}$. The temporal behavior of RESIK flare spectra has been analyzed for two different types of velocity distributions - a thermal (Maxwellian) distribution and a nonthermal plasma distribution of free electrons. The Si XIV, Si XIII, and Si XIId satellite lines observed with RESIK in the 5-6 A range were used to determine the degree of deviation from Maxwellian, and the equivalent non-Maxwellian pseudo-temperature, $\tau$. The diagnostics presented are sensitive to the shape of the distribution in the energy range where the maximum of the electron distribution occurs (where the bulk of electrons reside) and does not include the influence of the shape of the high-energy tail of the distribution. Under the assumption of a Maxwellian distribution of electron velocities, the plasma temperature was determined from an emission measure $(E M)$ loci analysis and a differential emission measure $(D E M)$ analysis of RESIK spectra. The high-energy end of the flare radiative emission was investigated through RHESSI spectral analysis.

Results. The nonthermal analysis of RESIK spectra has shown that the largest deviations of the plasma electron distribution from Maxwellian appeared during the impulsive phase of the flare. The decay phase spectra had an almost isothermal character. The pseudotemperature, $\tau$, reached its maximum around the peak time of the soft and hard X-ray fluxes. The temporal behavior of the temperatures derived from the thermal analysis was similar to the behavior of the nonthermal pseudo-temperature. The values of the pseudotemperature were consistent with the temperatures obtained in both thermal analyses, but lower than the temperatures derived from the slope of the RHESSI continua. In comparison with the synthetic isothermal or multithermal spectra, the nonthermal synthetic spectra fitted the observed Si XIId satellite lines much more closely (the error is less than 10\%). The fluxes in the Si XIId satellite lines in isothermal or multithermal spectra have been underestimated by a factor of three or more in comparison to the observed fluxes. The value of this factor varies with time and it is different for the different satellite lines.

Conclusions. Evidence was found for considerable deviations of the distribution of free electrons from Maxwellian in the plasma during a solar flare. These occurred mainly during the flare impulsive phase and can be diagnosed using existing X-ray spectral observations.
\end{abstract}

Key words. Sun: flares - Sun: X-rays, gamma rays

\section{Introduction}

Solar flare plasma originates under extreme physical conditions in the presence of strong magnetic fields and electric currents. Such conditions also result in the presence of nonthermal distributions of free electrons in the plasma. Only a few attempts to diagnose the shape of the electron distribution in solar flares from line spectra have so far been carried out. Seely et al. (1987) analyzed time dependent intensities of the Fe XXV resonance line and Fe XXIV satellite lines during several solar flares. Under the assumption of a nonthermal plasma distribution of electrons, Seely et al. (1987) found significant deviations from a Maxwellian distribution during the impulsive phase of the flares.
These nonthermal plasma electron distributions have higher and narrower peaks than the Maxwellian distribution, and can occur when energy is deposited into the tail of the distribution with a rate that is sufficiently high to overcome the processes that lead to equilibrium. The magnetic field increases the possibility of nonthermal plasma distributions because it inhibits the dissipation process. Dzif̌ćáková \& Karlický (2008) have shown that this kind of electron distribution in solar flare plasma can also be produced by the return current resulting from the high-energy electron beam that generates bremsstrahlung hard X-rays.

We have applied the new diagnostics of nonthermal plasma distributions proposed by Dzifčáková (2006a) to soft X-ray spectral observations. These diagnostics are sensitive to the 


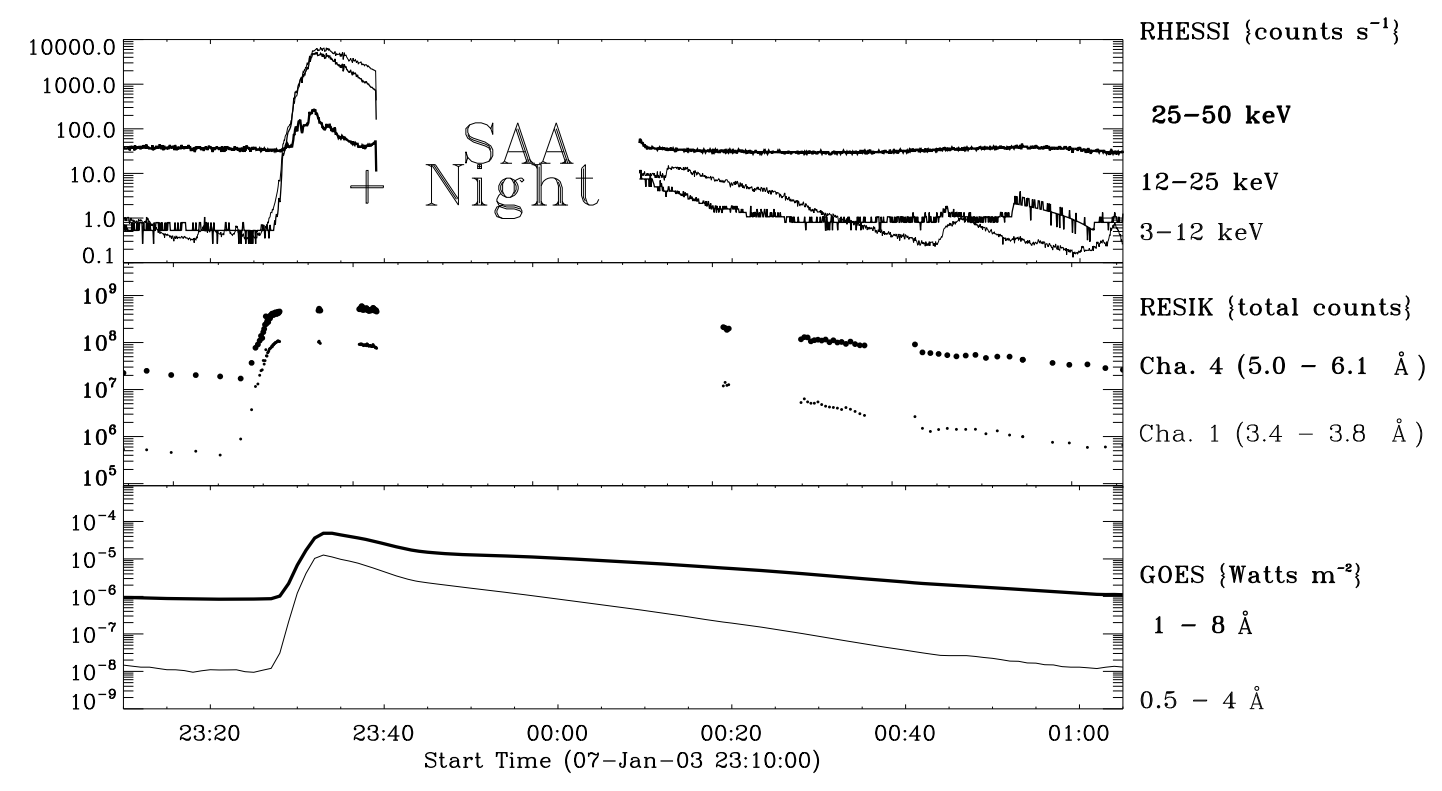

Fig. 1. Top panel: RHESSI lightcurves in the energy ranges 3-12, 12-25, and 25-50 keV averaged over RHESSI detectors 1, 3-6, 8, and 9. The peak of the high-energy nonthermal emission $(25-50 \mathrm{keV})$ is observed around 23:32 UT. The data gap between 23:40 and 00:10 UT is due to the spacecraft entering a South Atlantic Anomaly (SAA) period followed by night-time. Middle panel: RESIK integrated counts in the ranges 3.4-3.8 $\AA$ (channel 1) and 5.0-6.1 $\AA$ (channel 4). The data gap is due to night-time. Bottom panel: GOES fluxes in the $0.5-4 \AA$ and $1-8 \AA$ channels.

shape of the distribution in the energy region where the bulk of the electrons reside. They are not influenced by the shape of the high-energy tail of the electron distribution since its effect on the X-ray line spectrum is negligible. In this paper we will refer to this kind of nonthermal distribution as nonthermal plasma distribution. These results were then compared with the results obtained from isothermal and multithermal analyses. The goal of this paper is to determine whether it is possible to prove the occurrence of nonthermal plasma distributions in a flaring plasma from the available spectral data. For this analysis, we have selected a M4.9 class solar flare observed by the REngenovsky Spektrometr s Izgnutymi Kristalami (RESIK: Sylwester et al. 2005). RESIK is one of the instruments on board the Russian CORONAS-F mission, consisting of a high-resolution crystal spectrometer observing in the X-ray spectral range 3.4-6.1 $\AA$. The spectral range is divided into four channels (3.40-3.80 $\AA$, 3.83-4.27 $\AA, 4.35-4.86 \AA, 5.00-6.05 \AA$ ) and includes lines of He-like Ar, K, S and Si ions as well as associated dielectronic satellites. Therefore, RESIK can provide the information on soft X-rays between 2-4 keV (1st order) with a dispersion of 2.49-4.99 $\mathrm{m}^{\circ} \mathrm{bin}^{-1}$ (Sylwester et al. 2005). The impulsive phase and most of the flare decay were also observed by the Reuven Ramaty High Energy Solar Spectroscopic Imager (RHESSI: Lin et al. 2002). RHESSI samples the X-rays emission within the range $3 \mathrm{keV}$ up to $17 \mathrm{MeV}$ with a spectral resolution of 1 to $3 \mathrm{keV}$.

\section{Observations of the 2003 January $7 / 8$ flare}

According to the GOES observations, the M4.9 flare started on 2003 January 7 at 23:25 UT, reached the maximum at 23:33 UT, and ended on 2003 January 8 at 00:40 UT. The temporal evolution of the flare observed by RHESSI, RESIK and GOES is plotted in Fig. 1. We imaged the high energy emission in the $6-12 \mathrm{keV}$ soft $\mathrm{X}$-ray band, and in the $25-50 \mathrm{keV}$ hard X-ray band, using the CLEAN algorithm in the standard

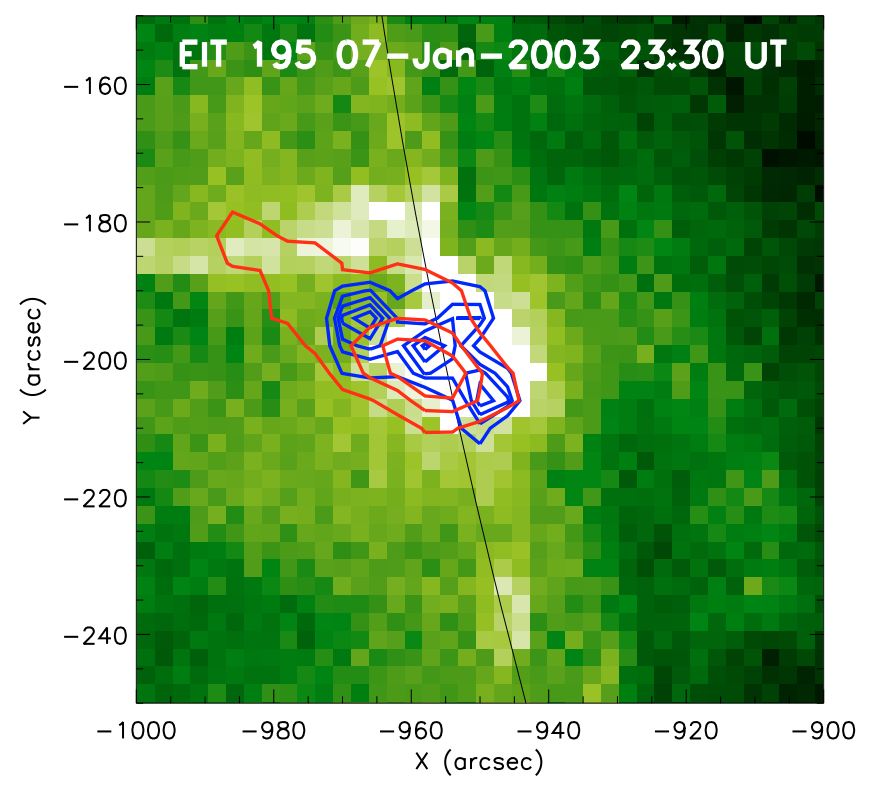

Fig. 2. RHESSI image contours in the 6-12 keV (red) and $25-50 \mathrm{keV}$ (blue) energy bands taken at approximately 23:30 UT. The contours were overplotted on a simultaneous EUV image seen by $\mathrm{SoHO} / \mathrm{EIT}$ in the $195 \AA$ filter. The solar limb is indicated.

RHESSI software (Fig. 2). At the flare peak (around 23:30 UT), a coronal source and footpoint sources can be observed in the 25-50 keV image contours. It is possible that some footpoint emission is occulted by the limb. The coronal source in the soft $\mathrm{X}$-ray band 6-12 keV is located at a different position.

\subsection{RESIK data reduction and processing}

The raw RESIK spectra were reduced to the absolute flux units by incorporating all known instrumental factors (Sylwester et al. 2005). 
Table 1. Time-averaged spectra during the 2003 January $7 / 8$ flare. The start and end times of intervals 0 to 15 , the number of spectra averaged in each interval and the total flux in all RESIK channels are indicated. The corresponding flare phases are also listed.

\begin{tabular}{ccccc}
\hline \hline Index & Time interval [UT] & Number of spectra & Total flux [ph s $\left.\mathrm{cm}^{2}\right]$ & Note \\
\hline 0 & $23: 13: 13-23: 21: 07$ & 5 & $1.22 \times 10^{5}$ & pre-flare \\
1 & $23: 26: 33-23: 28: 25$ & 1 & $1.09 \times 10^{5}$ & flare beginning \\
2 & $23: 28: 25-23: 28: 59$ & 2 & $3.46 \times 10^{5}$ & rise phase \\
3 & $23: 29: 17-23: 29: 57$ & 11 & $9.03 \times 10^{5}$ & rise phase \\
4 & $23: 30: 03-23: 30: 59$ & 14 & $2.37 \times 10^{6}$ & rise phase \\
5 & $23: 31: 03-23: 31: 47$ & 12 & $3.32 \times 10^{6}$ & rise phase \\
6 & $23: 36: 07-23: 36: 19$ & 4 & $3.84 \times 10^{6}$ & flare peak, polar region passby \\
7 & $23: 40: 39-23: 40: 59$ & 6 & $3.96 \times 10^{6}$ & flare peak, passby recovery \\
8 & $23: 41: 03-23: 41: 59$ & 15 & $3.67 \times 10^{6}$ & flare peak \\
9 & $23: 42: 03-23: 42: 35$ & 9 & $3.55 \times 10^{6}$ & flare peak \\
10 & $00: 21: 07-00: 21: 43$ & 4 & $1.23 \times 10^{6}$ & decay phase \\
11 & $00: 29: 41-00: 30: 03$ & 1 & $7.22 \times 10^{5}$ & decay phase \\
12 & $00: 30: 03-00: 30: 47$ & 3 & $7.50 \times 10^{5}$ & decay phase \\
13 & $00: 31: 09-00: 31: 57$ & 3 & $6.75 \times 10^{5}$ & decay phase \\
14 & $00: 32: 23-00: 32: 49$ & 2 & $6.39 \times 10^{5}$ & decay phase \\
15 & $00: 33: 15-00: 33: 41$ & 2 & $6.30 \times 10^{5}$ & decay phase \\
\hline
\end{tabular}

The averaged relative error of the data is $26 \%$. The observed spectra have been time-averaged as indicated in Table 1. Pre-flare spectra have also been included (time interval 0 ). The first degree polynomial fit to "line-free" sections of spectra in the RESIK channels 1 to $3(3.58-3.68 \AA, 3.83-3.93 \AA$, and 4.1-4.2 $\AA$, respectively) has been used for the subtraction of the continuum. There were no apparent lines in these ranges of RESIK spectra or in the synthetic spectra from CHIANTI. We therefore believe that this wavelength region shows real continuum.

The observed data after continuum subtraction show the residuals of the background flux (Fig. 3, top panel). We refer to each of these enhancements as "mounds". We have identified three mounds in each RESIK spectrum in the 3.4-6.1 range, centred respectively at the approximate wavelengths 4.4 , 5.2, and $5.95 \AA$. We assign this background flux enhancement to unknown instrumental or physical effects, since we observe it systematically in RESIK spectra. A similar portion of the solar X-ray spectrum was observed by the Bragg crystal spectrometer which was in orbit on the satellite OVI-17, launched on March 18, 1969 (Walker \& Rugge 1970). Their spectra cover the region of 3.75-8.5 $\AA$ and they did not observe any mounds. Therefore it is very likely that this effect has an instrumental origin and could be due to the fluorescence of the Al coating of an EUV blocking filter or to the Al crystal supports located at the crystal ends. Consequently, we remove the mounds from the spectra. The mounds were fitted by Gaussian functions using the xcfit.pro routine available in the SolarSoft package. The mound fits were then subtracted from the spectra (Fig. 3, bottom). The nonthermal and thermal diagnostics have been applied to the resulting spectra. Figure 3 (bottom) also shows the labels for the most important lines in the spectrum.

\section{The nonthermal plasma distribution}

We have assumed a nonthermal plasma distribution for the flaring plasma. The same kind of distribution has been used by Seely et al. (1987):

$f_{n}(\mathcal{E}) \mathrm{d} \mathcal{E}=\mathcal{B}^{n} \frac{2}{\sqrt{\pi}} \mathcal{E}^{\frac{n}{2}}(k T)^{-\left(\frac{n}{2}+1\right)} \exp (-\mathcal{E} / k T) \mathrm{d} \mathcal{E}$, where $\mathcal{B}^{n}=\frac{\pi^{1 / 2}}{2 \Gamma\left(\frac{n}{2}+1\right)}$ is the normalization constant, $\mathcal{E}$ is the energy of free electrons, $k$ is Boltzmann constant and $n$ and $T$ are the free parameters of the distribution. $T$ is not a thermodynamic temperature, despite the fact that it is also given in Kelvin. Parameter $n$ is dimensionless. The degree of deviation of a nonthermal plasma distribution from Maxwellian can be modeled by a free parameter $n$ (Fig. 4). For a Maxwellian distribution, $n=1$. The mean energy of the nonthermal plasma distribution depends on two parameters $n$ and $T$ :

$E_{n}=(n+2) k T / 2$.

In order to be able to compare the results with a thermal analysis, we have introduced a pseudo-temperature, $\tau$, which relates the mean energy of the nonthermal plasma distribution to the mean energy of the Maxwellian distribution (Dzifčáková 1998; Dzifčáková \& Kulinová 2001):

$E_{n}=\frac{3}{2} k \tau$

where $\tau$ is given in Kelvin. The relation between $\tau$ and $T$ is then

$\tau=\frac{n+2}{3} T$.

More information about this kind of nonthermal plasma distribution can be found in Hares et al. (1979), Seely et al. (1987), Dzifčáková (1998), Dzifčáková \& Kulinová (2001).

\section{Nonthermal diagnostics of RESIK observations}

The $\mathrm{Si}$ lines in the fourth RESIK channel covering the 5.00-6.05 A wavelength range (listed in Table 2) are suitable as diagnostics of nonthermal plasma distributions. However, the fluxes of these lines are affected by the presence of a large number of other lines. The set of Si synthetic spectra in the fourth RESIK channel have been computed for all known lines using version 5.2 of the SolarSoft CHIANTI package (Dere et al. 1997; Landi et al. 2006). CHIANTI routines have been specifically modified to assume a nonthermal plasma distribution of the free electrons (Dzifčáková 2006a). The values for parameter $n$ were $1,3,5,7,9,11$, while $\log (\tau)$ ranged between $6.7-7.3$ with a step 

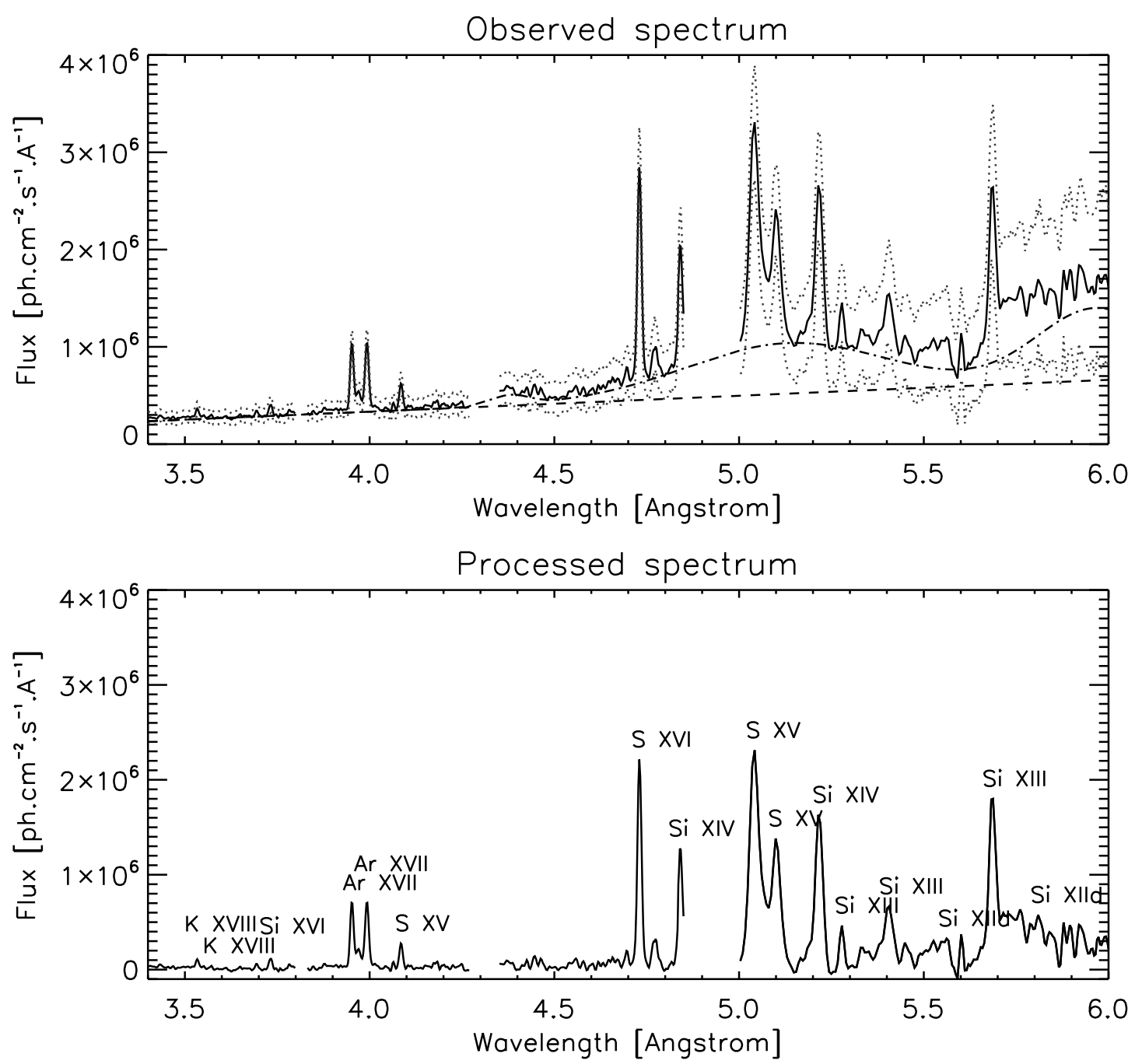

Fig. 3. Top panel: the observed RESIK spectrum in all four RESIK channels (separated by gaps) averaged over time interval 4 with overplotted continuum fit (dashed line) and "mounds" fit (dot-dashed line). The dotted lines show the error band of the data. Bottom panel: the resulting spectrum in all four RESIK channels after subtracting the continuum and fitted mounds. The most important lines are labeled.

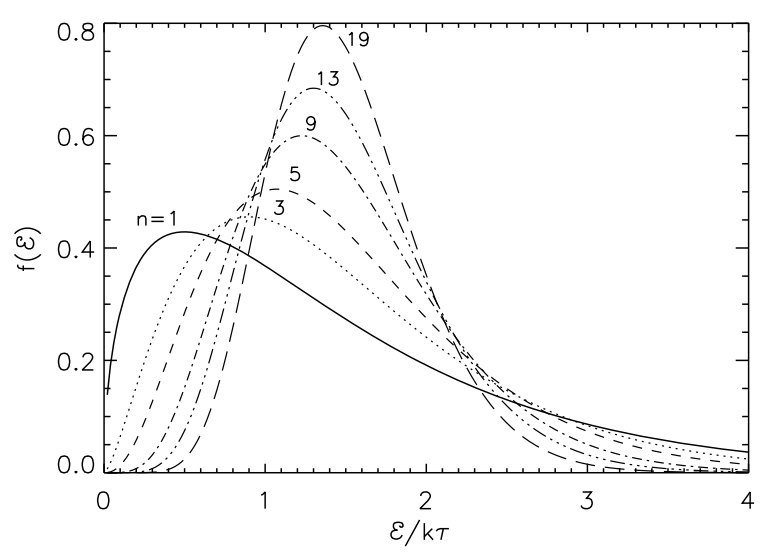

Fig. 4. Nonthermal plasma distributions as defined in Eq. (1). Although the shown distributions are of different shapes, they all have the same mean energy $E_{n}$. For a Maxwellian distribution is $n=1$. The nonthermal plasma distributions are labelled by their parameter $n=3,5,9$, 13, 19 and they have higher and narrower peaks with increasing $n$ in comparison with the Maxwellian distribution.

interval of 0.02. Several values for the full width at half maximum ( $F W H M)$, spanning the range of $F W H M$ measured for the lines in the RESIK observed spectra (24.0, 20.0 and $15.7 \mathrm{~m} \AA$ ) were used to calculate the synthetic spectra. The Si ionization equilibrium for the nonthermal plasma distributions was calculated by Dzifčáková (2005). Photospheric elemental abundances have been adopted for the computation of the synthetic spectra. We note that since we only computed synthetic lines from one element ( $\mathrm{Si}$ ), the abundance of elements has no effect on the diagnostics. A column emission measure of $10^{22} \mathrm{~cm}^{-5}$ has been assumed in the computation of all synthetic spectra.

The set of synthetic spectra for nonthermal plasma distributions has been calculated to obtain the theoretical dependence of the Si line ratios on the parameters of the nonthermal plasma distribution: $n$ and $\tau$. The lines of one ion that have a different behaviour of the excitation cross sections with energy or/and that are populated by different parts of the electron distribution can be sensitive to the presence of a nonthermal plasma distribution. The changes in the ratios of line intensities are usually small in this case. In general, the lines from different ionization stages are better as diagnostics, since the changes in the excitation equilibrium due to the nonthermal plasma distribution are coupled with changes in the ionization equilibrium. These line ratios are then more sensitive to the presence of the nonthermal plasma distribution (Dzifčáková 2006b; Dzifčáková \& Tóthová 2007). The 
Table 2. Spectral lines suitable as diagnostics of a nonthermal plasma distribution. The notation Si XIId denotes the satellite line excited by dielectronic recombination.

\begin{tabular}{cccc}
\hline \hline Line Number & Ion & Wavelength & Transition \\
\hline 1 & Si XIV & 5.22 & $1 \mathrm{~s}^{2} \mathrm{~S}_{1 / 2}-3 \mathrm{p}^{2} \mathrm{P}_{3 / 2,1 / 2}$ \\
2 & Si XIII & 5.28 & $1 \mathrm{~s}^{2}{ }^{1} \mathrm{~S}_{0}-1 \mathrm{~s} 5 \mathrm{p}{ }^{1} \mathrm{P}_{1}$ \\
3 & Si XIII & 5.40 & $1 \mathrm{~s}^{2}{ }^{1} \mathrm{~S}_{0}-1 \mathrm{~s} 4 \mathrm{p}{ }^{1} \mathrm{P}_{1}$ \\
4 & Si XIII & 5.68 & $1 \mathrm{~s}^{2}{ }^{1} \mathrm{~S}_{0}-1 \mathrm{~s} 3 \mathrm{p}{ }^{1} \mathrm{P}_{1}$ \\
5 & Si XIId & 5.56 & $1 \mathrm{~s}^{2} 2 \mathrm{p}^{2} \mathrm{P}_{1 / 2,3 / 2}-1 \mathrm{~s} 2 \mathrm{p} 4 \mathrm{p}{ }^{2} \mathrm{D}_{3 / 2,5 / 2}$ \\
6 & Si XIId & 5.82 & $1 \mathrm{~s}^{2} 2 \mathrm{p}^{2} \mathrm{P}_{1 / 2,3 / 2}-1 \mathrm{~s} 2 \mathrm{p}\left({ }^{3} \mathrm{P}\right) 3 \mathrm{p}^{2} \mathrm{D}_{3 / 2,5 / 2}$ \\
\hline
\end{tabular}

theoretical values of line ratios Si XIV (5.22 ̊̊)/Si XIII (5.68 $⿱$ ) vs. Si XIII $(5.68 \AA) / S i$ XIId $(5.82 \AA)$ for the particular parameter $n$ and the dependence of Si XIV (5.22 $\AA$ )/Si XIII (5.68 $⿱$ A) line ratio on $\log (\tau)$ are plotted as curves in Fig. 5. By overplotting the measured line ratios in the top panel of Fig. 5, the values of parameter $n$ can be estimated. Once the parameter $n$ is known, one can determine $\log (\tau)$ and thus the mean energy of the distribution using the plot in the bottom panel of Fig. 5. At present, we are not able to compute the continuum under the assumption of a nonthermal plasma distribution and to include it into a nonthermal analysis. Therefore, we have removed this continuum from the observed RESIK spectra, and we only measure the line fluxes.

The diagnostics for the shape of the nonthermal plasma distribution is based on the intensity ratios of Si lines (Dzifčáková \& Kulinová 2006). In principle, six Si lines (listed in Table 2) can be used for diagnosis. However, in principle, for reliable diagnosis we need:

- lines that have high intensities (to minimise the errors);

- lines for which the ratios have sufficient sensitivity to changes of the distribution shape (the changes due to the change of the distribution shape must be higher than the error in the determination of the line ratio);

- at least one satellite line must be used (satellite lines "sample" the electron distribution function).

Finally, only three Si lines observed in the fourth RESIK channel (5-6 $\AA$ ) fulfil these three conditions and therefore they were the

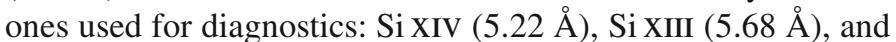
Si XIId (5.82 Å).

The satellite lines are very important for the nonthermal analysis of the shape of the distribution function because they sample the electron distribution at discrete energies. They are produced by dielectronic recombination which is a two-body process and only the electrons with energy within an autoionization width can recombine. The intensities of the allowed lines depend on the integral of the product of the collisional cross section and the electron velocity over the distribution function starting from the excitation energy. Thus, they are not so sensitive to the shape of the distribution. Therefore, the proposed diagnostics are very sensitive to the distribution shape in the energy range of the maximum of the distribution function and not very sensitive to the shape of the high energy tail of the distribution that produces the hard X-rays seen with RHESSI.

The xcfit.pro routine has been used to fit all spectral lines of sufficient strength to be resolved in the fourth RESIK channel. The spectral line profiles were assumed to be Gaussian functions and were fitted to achieve the smallest $\chi^{2}$ values. Some fifty Gaussians were needed to reproduce the whole spectrum from $5.15 \AA$ to $6.0 \AA$ with the precision on average better than $10 \%$. We have used $F W H M=15.7,20.0,24.0 \mathrm{~m} \AA$ and a variable $F W H M$ for the approximations. The best results were for
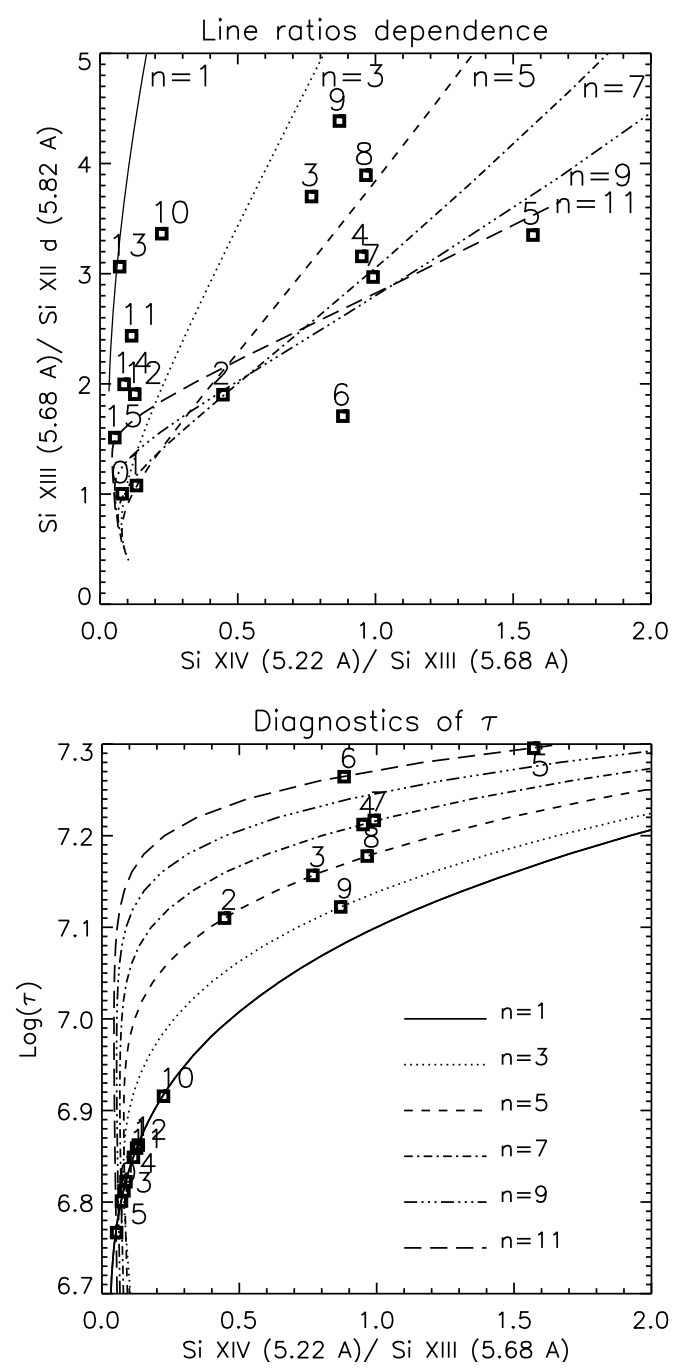

Fig. 5. Top panel: diagnostics of parameter $n$. The diagnostic curves for

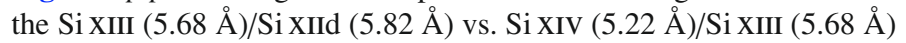
ratios calculated from synthetic spectra are overplotted with the points representing observed line ratios for the 16 time intervals listed in Table 1, for a fixed $F W H M=20.0 \mathrm{~m} \AA$ (squares). Line coding: full line $-n=1$, dot line $-n=3$, dash line $-n=5$, dot-dash line $n=7$, dot-dot-dot-dash line $-n=9$, long dash line $-n=11$. Bottom panel: diagnostics for $\tau$ and $E_{n}$. The dependence of the synthetic line ratio Si XIV (5.22 $\AA$ )/Si XIII (5.68 $\AA$ ) on $\tau$ is overplotted by the observed line ratio for the 16 time intervals and $F W H M=20.0 \mathrm{~m} \AA$. The line coding is the same as in the top panel.

variable $F W H M$ and for $F W H M=20.0 \mathrm{~m} \AA$. Then, the flux in each spectral line was calculated using the fitted amplitude of a Gaussian profile, central wavelength and FWHM. 

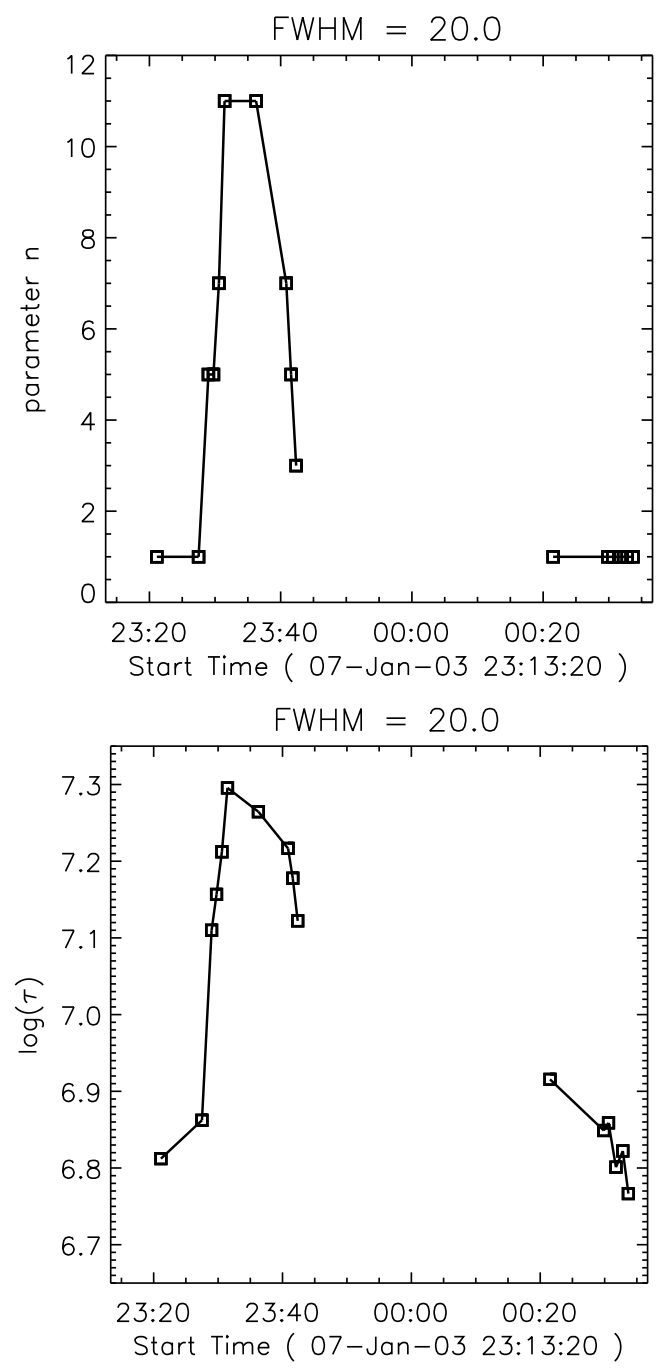

Fig. 6. Estimated parameter $n$ (top panel) and $\log (\tau)$ (bottom panel) for the 16 time-averaged spectra and for $F W H M=20.0 \mathrm{~m} \AA$.

The spectral Si XIV (5.22 $\AA$ ), and Si XIII (5.68 $\AA$ ) lines were the strongest lines in the spectral region between 5.15-6.0 $\AA$ which allowed us to fit them well (mean error 5\%). However, fitting the satellite lines Si XIId (5.56 $\AA$ ), and Si XIId (5.82 $\AA$ ) was more difficult (mean error $11 \%$ ) due to their low signal or the presence of other satellite lines in their vicinity. In general, the Si XIId (5.82 $\AA$ ) satellite line was more intense than the Si XIId (5.56 $\AA$ ) satellite line. Therefore, we only used the (5.82 $\AA$ ) line as a diagnostic of the parameters $n$ and $\tau$ for the nonthermal plasma distribution.

We have empirically determined the average $F W H M=$ $15.7 \mathrm{~m} \AA$ for the lines observed in the fourth RESIK spectral channel. This value was also used by Chifor et al. (2007). We have estimated a maximum $F W H M$ of $24.0 \mathrm{~m} \AA$ for the same RESIK channel. In our analysis, we considered $15.7 \mathrm{~m} \AA$ as the "instrumental width" value, i.e. the smallest width of the spectral line that can be measured in the fourth RESIK (5-6 $\AA$ ) channel. Besides the instrumental line broadening, the lines may broaden due to possible plasma motion or line blending. The thermal line width is much smaller than $15.7 \mathrm{~m} \AA$. In the fitting process we have allowed the $F W H M$ parameter to vary between $15.7 \mathrm{~m} \AA$ and $24.0 \mathrm{~m} \AA$. The average $F W H M$ of all fitted spectral lines in all 16 spectra in the fourth RESIK channel was then calculated to be $19.5 \mathrm{~m} \AA$. The mean $F W H M$ of the three fitted Si spectral lines used for nonthermal diagnostics was found to be $21.3 \mathrm{~m} \AA$. Finally, a constant $F W H M$ of $20.0 \mathrm{~m} \AA$ was then used for fitting all spectral lines in the fourth RESIK channel, in order to determine the dependence of the diagnosed plasma parameters on the empirically determined FWHM.

The line ratios obtained from the fitted $\mathrm{Si}$ lines of the 16 RESIK spectra are shown in Fig. 5. The results for constant FWHM are plotted together with the theoretical line ratios calculated from the synthetic spectra.

\subsection{Results from nonthermal analysis}

The nonthermal analysis results summarized in this section were obtained using the constant $F W H M$ line fitting. The results for spectra observed during time intervals 6 and 7 (Fig. 5) are influenced by the polar region passby of the satellite (Table 1). This means that these spectra could be contaminated by auroral emission and particles that were not properly removed. We note that these two spectra are the only spectra taken close to the flare peak phase but that they are less reliable. The evolution of the derived parameters $n$ and $\log \tau$ is plotted in Fig. 6. We found considerable deviations of the free electron distribution function from the Maxwellian distribution during the time interval 23:29-23:42 UT (time-averaged RESIK spectra indexed $3-9)$. The highest values of the parameter $n$ were obtained between 23:30-23:36 UT, for spectra indexed 4-7 $n \sim 11$ and $\tau \sim 2 \times 10^{7} \mathrm{~K}$ for spectrum 6). This interval coincides with the peak of the nonthermal hard X-ray (HXR) emission observed by RHESSI in $25-50 \mathrm{keV}$ energy range (Fig. 1). The RESIK spectra observed during the flare decay phase between 00:21-00:33 UT (indexed 10-15) appeared to be thermal (Fig. 13).

The results suggest that nonthermal plasma electron distributions are present in the flaring plasma during the impulsive phase, reaching the largest deviation from a Maxwellian distribution at around the peak of the X-ray flux, and starting to thermalize after the flare peak. This behaviour is in good agreement with the previous results from Seely et al. (1987). They found values of $n \sim 15-18$ and $T \sim 6 \times 10^{6}$ corresponding to $\tau=$ $T \cdot(n+2) / 3 \sim 4 \times 10^{7} \mathrm{~K}$ for the flares they studied. These values of $n$ and $\tau$ are higher than $n$ and $\tau$ in our flare. We have estimated the classification of the three solar flares analysed by Seely et al. (1987) from graphs of X-ray flux registered by GOES. One flare was X-class and the other two were high M-class events. The importance of our flare is M4.9. Therefore, if we suppose that stronger nonthermal effects are connected with the stronger $\mathrm{X}$-ray flux, then our lower values of $n$ and $\tau$ can be explained by the lower X-ray importance of the RESIK flare we analysed.

\section{Thermal diagnostics of RESIK observations}

The next two subsections describe the plasma diagnostic techniques we used to determine the thermal properties of the plasma: the $E M$ loci method and the differential emission measure $(D E M)$ analysis. The thermal diagnostics have been applied to the RESIK spectra in order to compare these results with those obtained with the nonthermal diagnostics. The lines in all four RESIK channels have been used for thermal diagnostics. They are labeled in Fig. 3 (bottom) and listed in Table 1 in Chifor et al. (2007). 

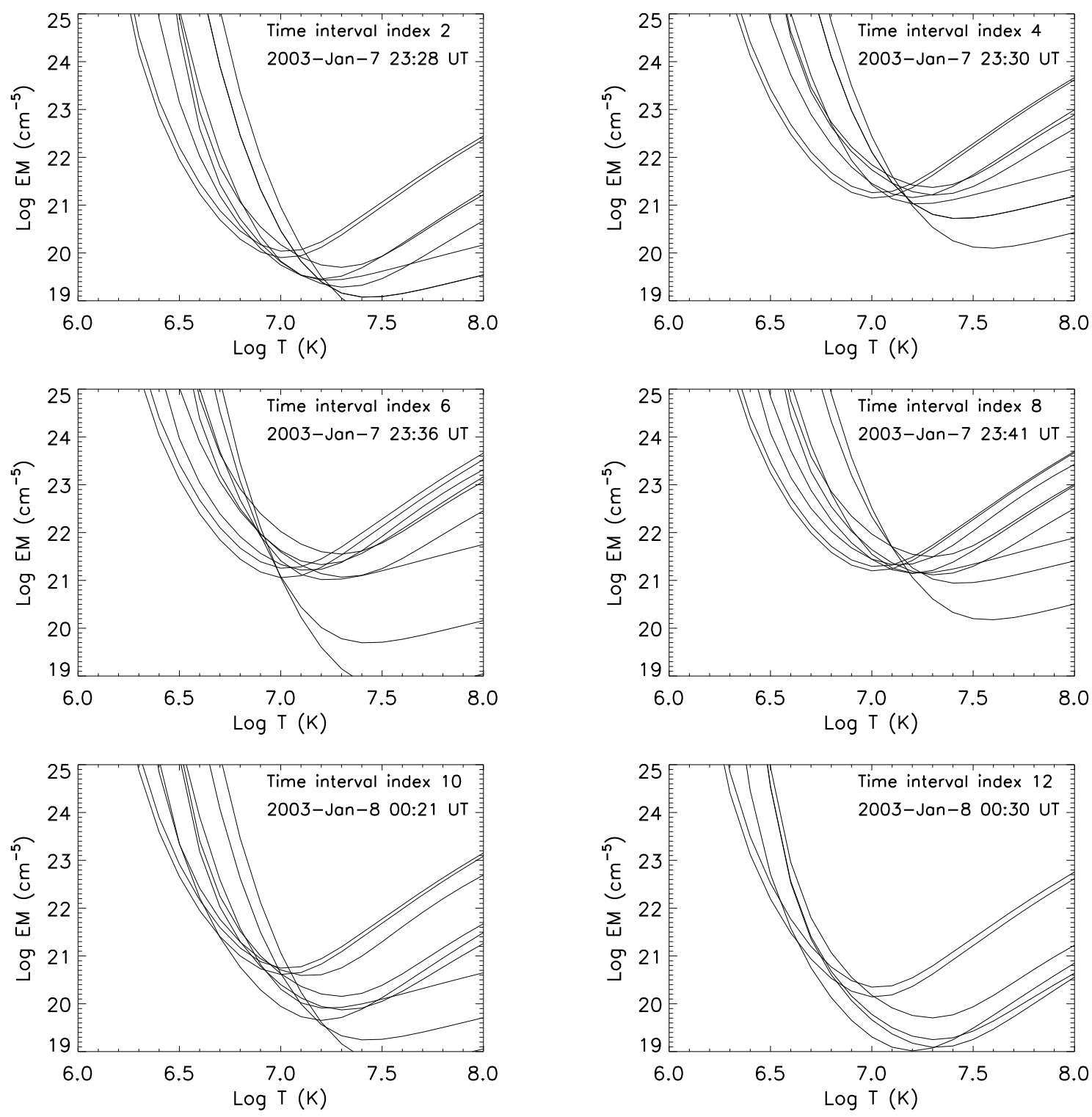

Fig. 7. Results from the line $E M$ loci analyses for time intervals 2, 4, 6, 8, 10, and 12 listed in Table 1.

\subsection{The EM loci method of analysis of RESIK spectral lines}

We performed an EM loci analysis (see e.g. Del Zanna et al. 2002; Chifor et al. 2007) on the observed RESIK line intensities. The flux observed at Earth (measured in photons $\mathrm{cm}^{-2} \mathrm{~s}^{-1}$ ) from an optically thin spectral line can be written as

$$
F=\frac{1}{4 \pi(1 \mathrm{AU})^{2}} \int G\left(T_{\mathrm{e}}, N_{\mathrm{e}}\right) N_{\mathrm{e}}^{2} \mathrm{~d} V
$$

where $T_{\mathrm{e}}$ and $N_{\mathrm{e}}$ are the electron temperature and density, $G\left(T_{\mathrm{e}}, N_{\mathrm{e}}\right)$ is the line contribution function, $V$ is the volume of the emitting plasma and $1 \mathrm{AU}$ is the Earth-Sun distance. From Eq. (4), the loci of the curves

$$
E M\left(T_{\mathrm{e}}\right)=4 \pi(1 \mathrm{AU})^{2} \frac{F}{G\left(T_{\mathrm{e}}, N_{\mathrm{e}}\right)}
$$

represent an upper limit to the emission measure distribution. For an isothermal plasma, all curves are expected to intersect at one point. An overlap between all the curves can also be used to validate the adopted relative elemental abundances.
Figure 7 shows results of the $E M$ loci analysis throughout the 2003 January 7/8 flare for the lines in all four RESIK channels listed in Chifor et al. (2007). We used ionization equilibrium data from Mazzotta et al. (1998). Photospheric abundances with depleted sulphur abundances, as in Chifor et al. (2007) were used to calculate the contribution functions for each observed emission line.

Under an isothermal approximation, the temperature increases from approximately $4 \times 10^{6} \mathrm{~K}(\log T=6.6 \mathrm{~K})$ at the beginning of the flare to $1.6 \times 10^{7} \mathrm{~K}(\log T=7.2 \mathrm{~K})$ during peak flare emission, and then decays to about $5 \times 10^{6} \mathrm{~K}(\log T=$ $6.7 \mathrm{~K})$. However, the intersection of the $E M$ curves only constrains the temperature to within \pm 0.2 in $\log T(\mathrm{~K})$, and, similar to Chifor et al. (2007), we concluded that the emitting flare plasma could be more accurately described by a multithermal distribution with one dominant temperature component.

\subsection{The DEM analysis of RESIK spectra}

The amount of emitting plasma within the instrument field of view (FOV) that has a temperature between $T$ and $T+\mathrm{d} T$ may 
be referred to as the differential emission measure $(D E M)$ of a multithermal plasma. The differential emission measure function $\operatorname{DEM}(T)$ is defined as

$\operatorname{DEM}(T)=N_{\mathrm{e}}^{2} \frac{\mathrm{d} V}{\mathrm{~d} T}$.

Assuming a constant abundance within the instrument FOV, the intensity of the observed line flux from multithermal plasma is given by

$F=\frac{1}{4 \pi(1 \mathrm{AU})^{2}} \int G\left(T_{\mathrm{e}}, N_{\mathrm{e}}\right) \operatorname{DEM}(T) \mathrm{d} T$.

To evaluate the $\operatorname{DEM}(T)$ distribution, we used an application of the "maximum entropy method" implemented by Del Zanna (1999). This method has been employed in the analysis of RESIK spectra by Chifor et al. (2007) who give a description of the application.

We calculated DEM distributions (Fig. 8) using the fitted line fluxes in all 4 RESIK channels (Chifor et al. 2007), together with fitted continuum fluxes in $0.2 \AA$ wide bands. After finding an appropriate $D E M$ function in each time interval, synthetic spectra in the RESIK wavelength range were then calculated. By comparing the synthetic spectra with the observed spectra in all RESIK channels, we obtained the same set of chemical abundances found in Chifor et al. (2007), namely a photospheric (Asplund et al. 2005) abundance for Si and Ar, and a depleted $\mathrm{S}$ abundance. The uncertainty in the measurement is mostly due to a relatively small $(30 \%)$ uncertainty in the intensities of the $\mathrm{Si}, \mathrm{Ar}, \mathrm{S}$ lines, and a (difficult to estimate) uncertainty in the theoretical emissivity of the lines, mostly due to the ionisation and recombination rates affecting the ion populations.

The relative intensities of the RESIK Si and Ar lines (in the present study as well as in Chifor et al. 2007) are very well represented using of the latest photospheric abundances (Asplund et al. 2005). On the other hand, the RESIK S abundance was found to be lower than the photospheric value by a factor of $\sim 2$. This value $\left(7.9 \times 10^{-6}\right)$ is the same value found for $S$ by Fludra \& Schmelz (1999), who derived the absolute abundances of $\mathrm{S}, \mathrm{Ca}$ and $\mathrm{Fe}$ for 57 flares observed with the Bragg Crystal Spectrometer (BCS) on Yohkoh. Fludra \& Schmelz (1999) reported a large scatter in the abundance of Fe, finding a slightly higher than accepted photospheric value. The RESIK S abundance value is also close to the value previously found by Fludra \& Schmelz (1995) for the 1980 August 25 SMM flare $(9.7 \times$ $\left.10^{-6}\right)$. The RESIK Si abundance $\left(3.2 \times 10^{-5}\right)$ is very close to the value found by the same authors for the 1980 November 5 SMM flare $\left(3.3 \times 10^{-5}\right)$. We note, however, that in the extended literature on elemental abundance measurements during flares, a large scatter of values can be found. We also note that if a further instrumental background component were present, it would affect our results in terms of an increase in the elemental abundances. If we removed any additional "pedestal" from channels 3 and 4 of RESIK, we would have disagreement between the DEMs estimated from the line and continuum fluxes for the post-impulsive phases. The fact that the calibrated SMM and Yohkoh spectra produced very close values for the abundances derived from lineto-continuum ratios suggests that the RESIK calibration is valid.

The DEM analysis indicates a multithermal nature of the emitting plasma, confirming the presence of a dominant temperature component peaking close to the temperatures at which the RESIK line $E M$ curves intersected in the $E M$ loci analysis.

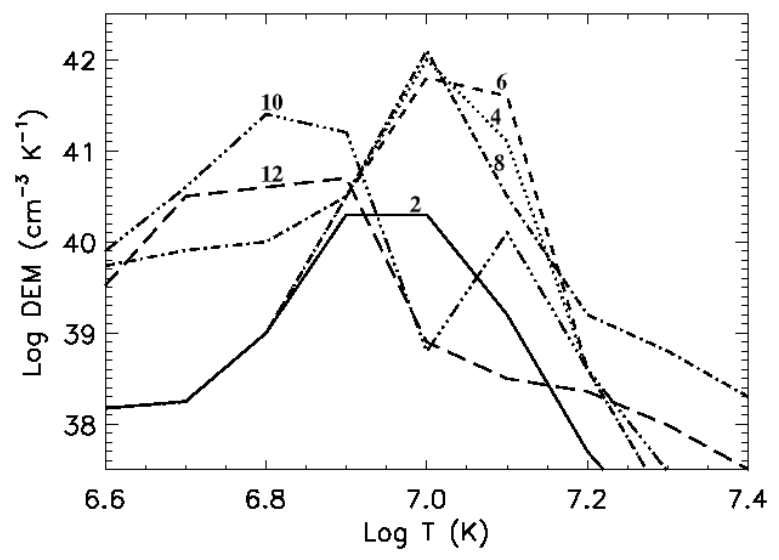

Fig. 8. $D E M$ distributions calculated throughout the 2003 January $7 / 8$ flare from the observed RESIK lines and continua. The time indeces relating to the time intervals given in Table 1 are indicated as following: full line -2 , dot line -4 , dash line -6 , dot-dash line -8 , dot-dot-dotdash line -10 , long dash line -12 . We used these calculated DEM distributions to produce CHIANTI synthetic intensities which were compared with the observed RESIK spectra.

\section{RHESSI spectral analysis}

We further analyzed the high-energy end of the 2003 January $7 / 8$ flare emission using RHESSI data in the time intervals used in the RESIK analysis during which RHESSI observations were available (time intervals $2-6$ and $10-15$ of Table 1). RHESSI spectra were fitted by means of the Object Spectral Executive (OSPEX) package which uses the CHIANTI atomic code. The default set of abundances in OSPEX is represented by the solar coronal elemental abundances from Feldman et al. (1992). We modified this default option to employ the same set of photospheric abundances used for the RESIK spectra (the dominant contribution being from $\mathrm{Fe}$ and $\mathrm{Ni}$ ). We note that only the iron abundance is relevant for RHESSI and the difference between the default coronal abundances and the photospheric abundance we assumed is a factor of four. A sample RHESSI spectral fit between 23:30:22 UT and 23:30:30 UT (corresponding to RESIK time interval 3) is given in Fig. 9. We modeled the thermal plasma emission with an isothermal component and 3 Gaussian functions fitting the $\mathrm{Fe}$ and $\mathrm{Fe} / \mathrm{Ni}$ line complexes and a residual instrumental line at $\sim 10 \mathrm{keV}$ (see Phillips et al. 2006, for an account of the RHESSI line features). The nonthermal power-law high-energy emission, conspicuous during the 25-50 keV HXR bursts and noticeable in RHESSI lightcurves, was modeled with a thick-target bremsstrahlung component from a power-law electron distribution with a low-energy cutoff at $27 \mathrm{keV}$. Figure 9 also shows that the emission from the power-law high-energy tail of the photon spectrum above $\sim 20 \mathrm{keV}$ is more than one and a half orders of magnitude lower than the total X-ray emission in the spectral region below $6 \mathrm{keV}$ where RESIK observes. Using RHESSI soft X-ray flare spectra, Dennis et al. (2005) and Phillips et al. (2006) found coronal Fe abundance. However, Phillips et al. (2006) used flare data from long duration flares, during the decay phase only. In this paper (as in Fludra \& Schmeltz 1999) we focus on the impulsive phase of the flare (when nonthermal effects are likely to be highest). The difference in the temperatures we derived from the slope of the fitted RHESSI thermal continuum using photospheric vs. coronal abundances is at most $1 \mathrm{MK}$. 


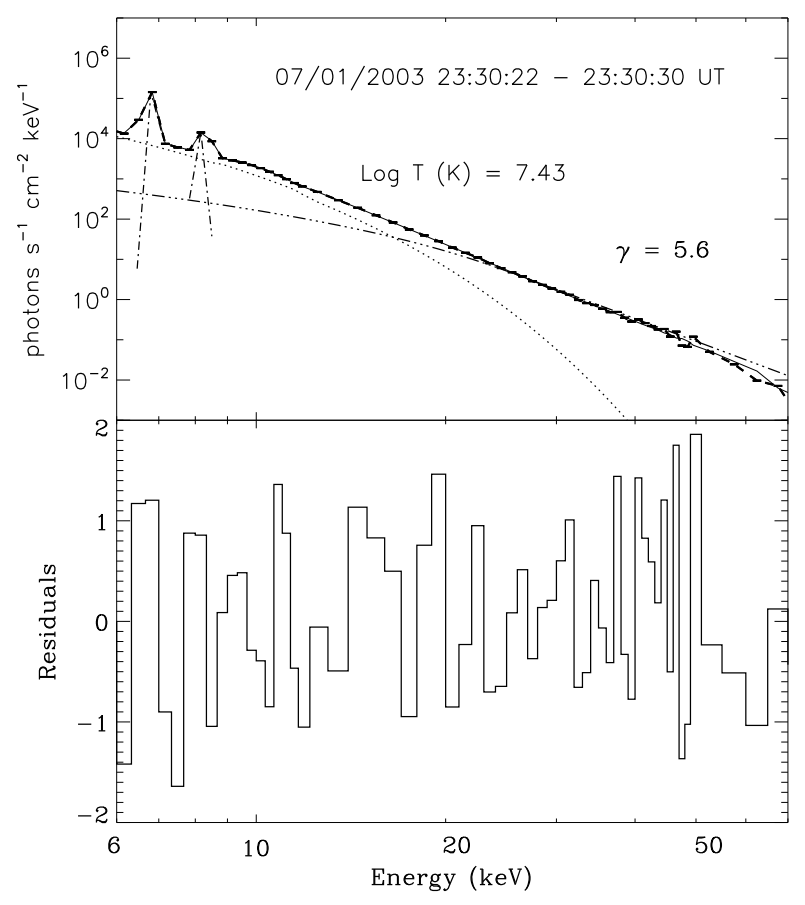

Fig. 9. Upper panel: RHESSI spectrum (dashed curve) accumulated between 23:30:22 and 23:30:30 UT. The thermal continuum was modelled with one isothermal component (dotted curve). The fitted $\mathrm{Fe}$ and $\mathrm{Fe} / \mathrm{Ni}$ features are also shown (dot-dashed curves). The high-energy tail of the spectrum was fitted with a thick-target bremmstrahlung component (dashed dot-dot) with the lower cutoff energy of the power-law electron spectrum of $27 \mathrm{keV}$. The power-law index of the photon spectrum $\gamma$ is 5.6. The sum of all models (best-fit model) is represented by the continuous curve. For a fitting range was $6-70 \mathrm{keV}$. Lower panel: fit residuals representing the number of standard deviations of the best-fit model above or below data. The reduced $\chi^{2}$ for the quality of fit was 1.0 .

\section{Comparison of results using the different diagnostics}

Figure 10 shows the evolution of plasma temperature $T$ derived as each of the following:

- the pseudo-temperature $\tau$ derived from the nonthermal diagnostics of plasma kernel;

- temperature derived from the ratio of the two GOES channels, assuming an isothermal plasma;

- estimated $T$ from the line $E M$ loci analysis, under an isothermal approximation;

- $T$ corresponding to the maximum of the computed $D E M$ functions;

- $T$ derived from the fit to the RHESSI X-ray spectra above $6 \mathrm{keV}$ assuming an isothermal component and a nonthermal electron distribution.

We find overall good agreement between the temperatures derived with the different methods. However, the temperatures obtained from RHESSI continua appear higher during the peak phase of the flare. This may suggest a non-isothermal nature of flare plasma around the flare peak. It is likely that RHESSI picked up a higher temperature component during these times, since its detectors are sensitive to higher energy emission than RESIK or GOES. The Fe and Fe/Ni features at $\sim 6.7 \mathrm{keV}$ and $\sim 8 \mathrm{keV}$ are most conspicuous in RHESSI spectra at the flare peak and shortly after, and the presence of both of them is evidence for temperatures $\gtrsim 20 \mathrm{MK}$ (Phillips et al. 2006).

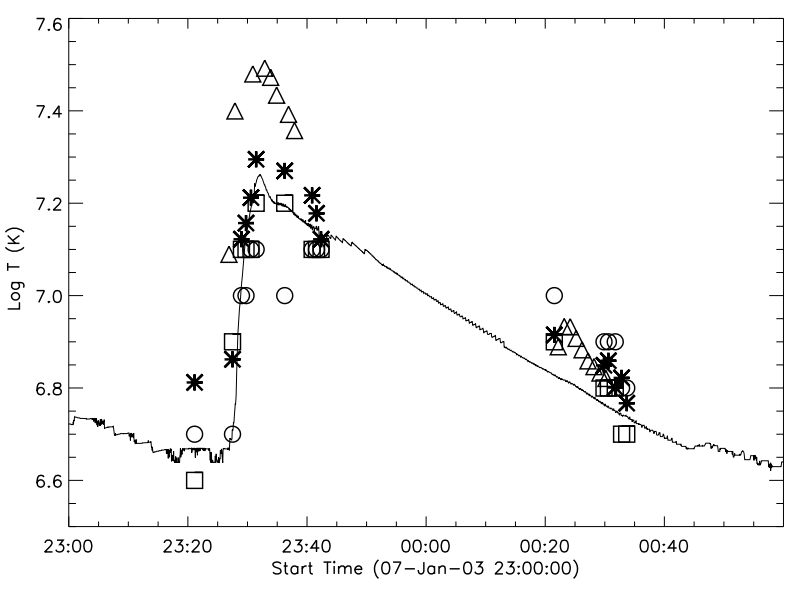

Fig. 10. Evolution of temperature during the 2003 Jan. 07/08 flare: the equivalent temperature, $\tau$ derived from nonthermal analysis (stars), $T$ derived from the line $E M$ loci analysis (squares), $T_{\max }$ corresponding to the maximum for the DEM curves (circles), $T$ calculated from the ratio of the two GOES channels assuming isothermal plasma (solid curve), and $T$ derived from the slope of fitted RHESSI continua (triangles).

The chief aim of the analysis presented in this paper is to diagnose the occurrence of nonthermal plasma electron distributions in solar flare plasma using X-ray observations taken with the RESIK instrument. For this purpose, both nonthermal and thermal diagnostics have been applied to the observed flare spectra.

In spite of the good agreement between the pseudotemperature, $\tau$, obtained from the nonthermal analysis, and the temperatures derived with the different thermal diagnostics (e.g. the $E M$ loci and the $D E M$ analyses) the characteristics of the synthetic spectra are different. When the flare spectra are assumed to be either isothermal or multithermal, the fluxes of the Si satellite lines observed by RESIK are underestimated. The nonthermal plasma distribution assumption provides higher intensities for the observed satellite lines as well as matching the observed ratio of Si XIV 5.22 ̊/Si XIII $5.68 \AA$.

In order to compare the results from the nonthermal and thermal analyses, we compared the observed spectra with the synthetic spectra computed under the assumption of a (a) nonthermal; (b) multi-thermal; and (c) isothermal plasma, respectively (Figs. 11 and 12). We note that except for the known lines, the observed spectra may also contain unknown spectral lines in the 5-6 $\AA$ range which could not be included into the computed synthetic spectra due to a lack of atomic data in the literature and in CHIANTI. Therefore, we can only compare the observed and synthetic fluxes of the identified lines. Synthetic spectra calculated under the assumption of a nonthermal plasma distribution matched the observed spectra better than the isothermal synthetic spectra. This is particularly conspicuous for time interval 4 (Fig. 12), where the DEM analysis shows the presence of a sharp maximum, and plasma could be close to isothermal. The synthetic fluxes of the allowed lines of Si XIV $5.22 \AA$ and Si XIII $5.68 \AA$ agree well with the observed ones for both nonthermal and isothermal assumptions. However, for spectra 2-9, the Si XIId $5.56 \AA$ and $5.82 \AA$ satellite lines for a isothermal approximation were smaller by a factor of three or more than the observed satellites. The value of this factor varies for the different satellite lines. The spectra from the decay phase had an isothermal character and could be approximated by a Maxwellian isothermal synthetic spectrum (Fig. 13). 


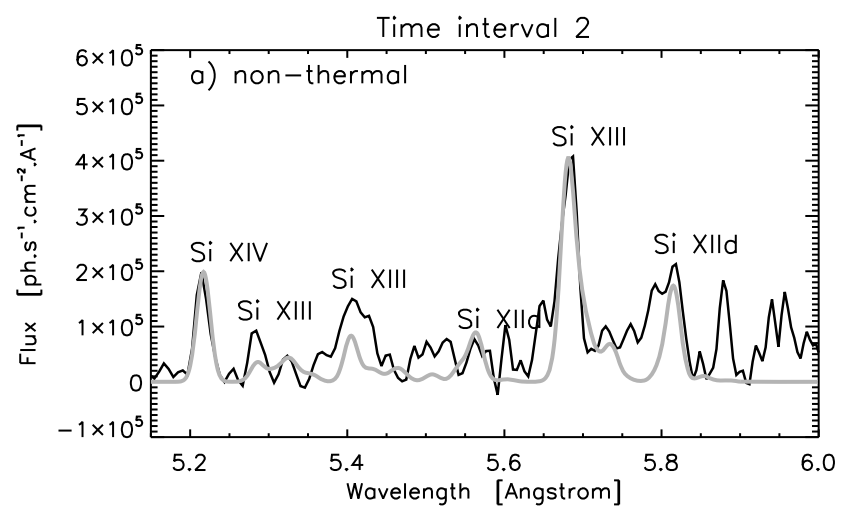

Time interval 2

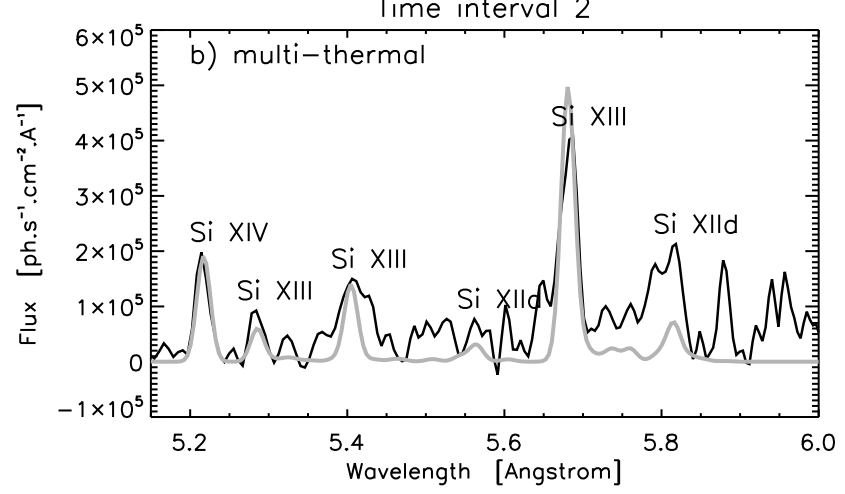

Time interval 2

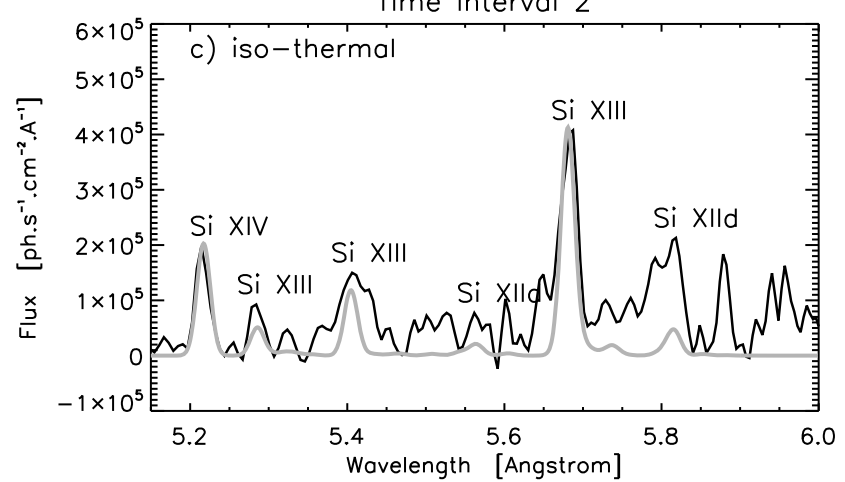

Fig. 11. Comparison of the observed spectra at time interval 23:28:25-23:28:59 UT (black curve), a) with the synthetic spectrum (grey curve) calculated under the assumption of the nonthermal plasma distribution with $n=5, \log (\tau)=7.122$ and for $F W H M=20 \mathrm{~m} \AA$, and $\left.E M=6.6 \times 10^{20} \mathrm{~cm}^{-5} ; \mathbf{b}\right)$ with the multithermal synthetic spectrum (grey curve) calculated using the derived $D E M$ and c) with an isothermal synthetic spectrum (grey curve) calculated under the assumption of a Maxwellian distribution with the same value of the line ratio Si XIV (5.22 $\AA$ )/Si XIII (5.68 $\AA$ ) as in a) and for $\log (T)=7.00$ and $E M=9.8 \times 10^{20} \mathrm{~cm}^{-5}$.

\section{Discussion}

The nonthermal synthetic spectra are able to fit the satellite lines much better than the isothermal or multithermal synthetic spectra, and are also able to reproduce the observed fluxes of the allowed lines Si XIV 5.22 $\AA$ and Si XIII 5.68 $\AA$. This was not achievable for the assumption of a Maxwellian distribution. Nevertheless, the nonthermal synthetic spectra failed to reproduce the Si XIII 5.28 ̊ and $5.40 \AA$ line fluxes. The Si XIII $5.28 \AA$ line flux was very low for higher $\tau$ when the distribution appeared to be more nonthermal. However, the fluxes of both lines are also underestimated for an iso-thermal Maxwell distribution
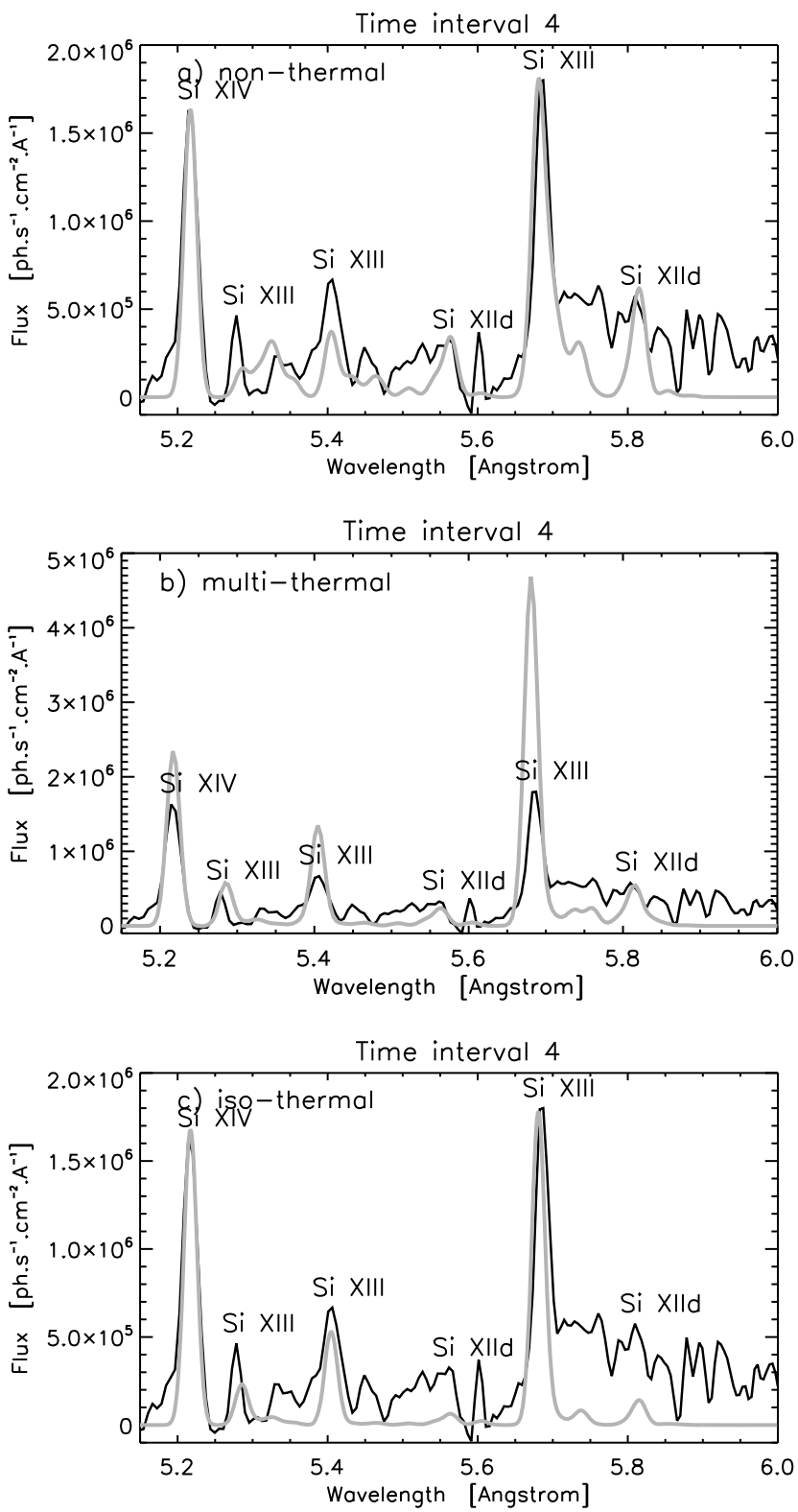

Fig. 12. Comparison of the observed spectra at time interval 23:30:03-23:30:59 UT (black curve, a) with the synthetic spectrum (grey curve) calculated under the assumption of the nonthermal plasma distribution with $n=7, \log (\tau)=7.212$ and for $F W H M=20 \mathrm{~m} \AA$ and $E M=3.8 \times 10^{21} \mathrm{~cm}^{-5}$; b) with the multithermal synthetic spectrum (grey curve) calculated using the derived $D E M$ and c) with an isothermal synthetic spectrum (grey curve) calculated under the assumption of a Maxwellian distribution with the same value of the line ratio Si XIV $(5.22 \AA) / \operatorname{Si~XIII~}(5.68 \AA)$ as in a) and for $\log (T)=7.1$ and $E M=4.7 \times 10^{21} \mathrm{~cm}^{-5}$.

(Figs. 11c, 12c). These lines belong to the higher members of line series for which Kepa et al. (2006) detected higher fluxes during the impulsive phase of flares observed by RESIK. The best results for these lines are obtained under a multithermal approximation (Fig. 11b). It is possible that a multicomponent nonthermal plasma approximation could also reproduce the flux in this line much better than a one-component nonthermal approximation. The real flaring plasmas should be naturally inhomogeneous. A multicomponent nonthermal analysis would require too many free parameters to be fitted, and cannot be carried out at 


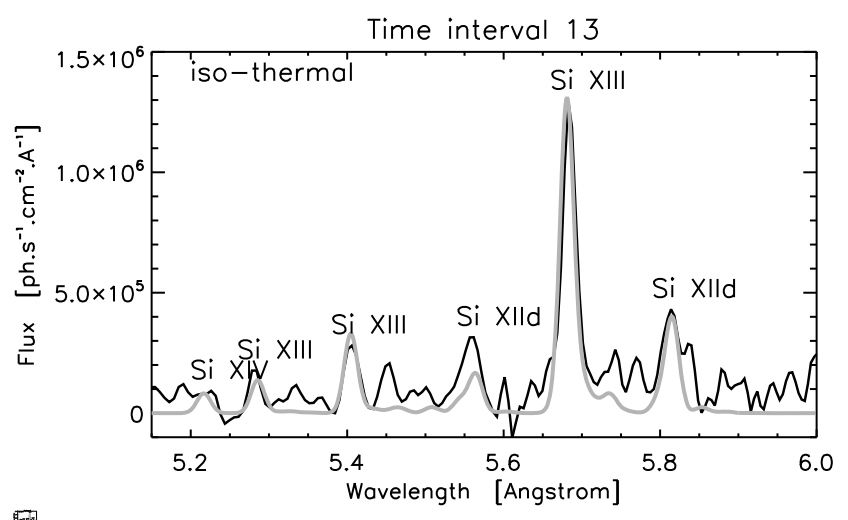

Fig. 13. Comparison of the observed spectra at time interval 00:31:09-00:31:57 UT (black curve) with the synthetic spectrum (grey curve) calculated under the assumption of a Maxwellian distribution with $\log (T)=6.8, F W H M=20 \mathrm{~mA}$ and $E M=7.0 \times 10^{21} \mathrm{~cm}^{-5}$.

present. A multicomponent nonthermal analysis of RESIK spectra is intended for the future.

We note that there are several sources of errors affecting our results. The first one is the presence of the "mounds" in spectra. The third mound centered around $5.95 \AA$ is located at the end of the fourth channel where the Si XIId satellite lines are observed. Therefore the fit of this mound strongly influences the intensity of the Si XIId $5.82 \AA$ line.

In our nonthermal diagnostics we have used theoretical line fluxes calculated for a constant $F W H M$. The $F W H M$ should be same for all lines of one element that originate under the same physical conditions, and they should be identically broadened due to instrumental effects. We found the instrumental broadening is much larger than the other possible effects. Nevertheless, the lines in the observed spectrum do not have the same FWHM. This affects the determination of line fluxes and the estimate of the parameter, $n$, that indicates the degree of deviation from a Maxwellian distribution. However, data errors and the blending of the lines can partly account for this effect. This problem introduces an error of about $10 \%$ in the nonthermal analysis.

The fluxes of spectral lines, mainly Si XIId (5.82 A) and Si XIII (5.68 $\AA$ ), are affected by the presence of blends. For example, in the vicinity of Si XIII (5.68 $\AA$ ) there are more than 20 fainter Si XIId satellite lines. The importance of these satellites increases with an increase of the parameter $n$. The fluxes of satellite lines have been included in the synthetic fluxes of the lines which were used as diagnostics. In the vicinity of the three diagnostic lines, there are also the lines of Al XIII, P XIV, P XV and S XIVd satellite lines but they have very low fluxes.

By assuming the presence of a nonthermal plasma distribution of free electrons, one is able to reproduce the observed fluxes of the satellite lines; this is not possible in the isothermal or multithermal cases. This result provides evidence for nonthermal plasma distributions in flares, despite the fact that not all features in X-ray spectra could be reproduced. Future analyses of flare observations in other spectral ranges may be required in order to confirm the presence of the nonthermal plasma distributions indicated by our analysis.

\section{Conclusion}

A nonthermal analysis of X-ray spectra observed by the RESIK spectrograph during an M4.9 flare has indicated the presence of nonthermal plasma distributions of free electrons. The plasma appears to be nonthermal during the impulsive phase of the flare, while the highest deviations of the electron distribution from the Maxwellian distribution were found at the flare peak, coincident with a HXR burst observed by RHESSI. The comparison of thermal and nonthermal analyses has indicated good agreement between the thermal temperature derived with the different methods and its equivalent, the pseudo-temperature, derived from a nonthermal analysis.

Although uncertainties in the Si line spectra observed with RESIK are high, we have shown that it is possible to directly probe the nonthermality of the solar plasma distribution using X-ray observations. Considering that the majority of flare plasma models assume thermal distributions, the fact that we have found evidence for nonthermal plasma distributions in solar flare plasma is significant.

Acknowledgements. This work has been supported by the Scientific Grant Agency VEGA, Slovakia, grant No. 1/0069/08. We are very thankful for the open data policy of RESIK, RHESSI and GOES. CHIANTI is a collaborative project involving project involving the Naval Research Observatory and George Mason University (USA), the Rutherford Appleton Laboratory, the Mullard Space Science Laboratory and University of Cambridge (UK) and the University of Florence (Italy). C.C. is grateful for scholarship support received from the University of Cambridge Overseas Trust, an Isaac Newton Studentship from the Cambridge Institute of Astronomy and an Overseas Research Student Award. G.D.Z. thanks STFC for its support and DAMTP for its hospitality. H.E.M. acknowledges the support of STFC. B.S. and J.S. acknowledge support from the Polish Ministry of Science grant 1.P03D.017.29. The work was facilitated by a Royal Society travel grant which enabled ED to visit HEM's group during the summer of 2006.

\section{References}

Asplund, M., Grevesse, N., \& Sauval, A. J. 2005, ASPC, 336, 25 Chifor, C., Del Zanna, G., Mason, H. E., et al. 2007, A\&A, 462, 323 Del Zanna, G. 1999, Ph.D. Thesis, Univ. of Central Lancashire Del Zanna, G., Landini, M., \& Mason, H. E. 2002, A\&A, 385, 968 Dennis, B. R., Phillips, K. J. H., Sylwester, J., et al. 2005, AdSpR, 35, 1723 Dere, K. P., Landi, E., Mason, H. E., Monsignori Fossi, B. C., \& Young, P. R. 1997, A\&AS, 125, 149

Dzifčáková, E. 1998, Sol. Phys., 178, 317

Dzifčáková, E. 2005, Proc. of the 11th European Solar Physics Meeting, The Dynamic Sun: Challenges for Theory and Observations, 11-16 September, Leuven, Belgium, ed. D. Danesy, S. Poedts, A. De Groof, \& J. Andries, Published on CDROM, ESA SP-600, 119

Dzifčáková, E. 2006a, Proc. of SoHO-17: 10 Years of SoHO and Beyond, 7-12 May, Giardini Naxos, Sicily, Italy, ed. H. Lacoste, \& L. Ouwehand, ESA SP-617, 89.1

Dzifčáková, E. 2006b, Sol. Phys., 234, 243

Dzifčáková, E., \& Kulinová, A. 2001, Sol. Phys., 203, 53

Dzif̌cáková, E., \& Karlický, M. 2008, Sol. Phys., submitted

Dzifčáková, E., \& Kulinová, A. 2006, Proc. of SoHO-17: 10 Years of SoHO and Beyond, 7-12 May, Giardini Naxos, Sicily, Italy, ed. H. Lacoste, \& L. Ouwehand, ESA SP-617, 88.1

Dzif̌cáková, E., \& Tóthová, D. 2007, Sol. Phys., 240, 211

Feldman, U., Mandelbaum, P., Seely, J. L., Doschek, G. A., \& Gursky, H. 1992, ApJS, 81, 387

Fludra, A., \& Schmelz, J. T. 1995, ApJ, 447, 936

Fludra, A., \& Schmelz, J. T. 1999, A\&A, 348, 286

Hares, D. J., Kilkenny, J. D., Key, M. H., \& Lunney, J. G. 1979, Phys. Rev. Lett., 42, 1216

Kepa, A., Sylwester, J., Sylwester, B., et al. 2006, AdSpR, 38, 1538

Landi, E., Del Zanna, G., Young, P. R., et al. 2006, ApJS, 162, 261

Lin, R. P., Dennis, B. R., Hurford, G. J., \& 65 co-authors 2002, Sol. Phys., 210,

Mazzotta, P., Mazzitelli, G., Colafrancesco, S., \& Vittorio, N. 1998, A\&AS, 133, 403

Phillips, K. J. H. Chifor, C., \& Dennis, B. R. 2006, ApJ, 647, 1480

Seely, J. F., Feldman, U., \& Doschek, G. A. 1987, ApJ, 319, 541

Sylwester, J., Gaicki, I., Kordylewski, Z., et al. 2005, Sol. Phys., 226, 45

Walker, A. B. C., Jr., \& Rugge, H. R. 1970, A\&A, 5, 4 